\title{
Morbid obesity in South Africa: considerations and solutions
}

\author{
JA Dave \\ Division of Endocrinology, Groote Schuur Hospital, University of Cape Town, South Africa
}

Corresponding author, email: joeldave@endocrine.co.za

The global obesity epidemic has not spared South Africa, making it relevant to report results of metabolic surgery performed in South Africa. In this issue the outcomes of a single surgeon experience in the private sector with laparoscopic gastric banding is presented. ${ }^{1}$ Before addressing the surgical aspects of obesity, it is important to give context to the epidemic. Since 1975, the numbers of obese people in the world have tripled with more than 1.9 billion adults (> 18 years old) overweight or obese (609 million in 2015). ${ }^{2}$ More concerning is the 41 million children $(<5$ years old $)$ and 340 million children or adolescents (5-16 years old) reported in 2016 to be either overweight or obese. Over the past 2 decades, overweight and obesity has been increasing in Africa, more so in women than in men. ${ }^{2}$ In South Africa, there were an estimated 19 million overweight or obese adults in 2016 and in $201220 \%$ of children (2-5 years old) were estimated to be overweight or obese. ${ }^{3,4}$ The morbidity and mortality associated with obesity is well documented, as is the improvement in all associated comorbidity with weight loss. ${ }^{5,6}$ The pathophysiology of obesity is complex, including signals from multiple organ systems all channelled through the hypothalamus thereby affecting appetite. This makes the management of obesity difficult. Numerous guidelines exist globally, and, although they differ in their scope of guidance, they are all in agreement that lifestyle modification should form the foundation of any management plan for obesity. However, few patients are able to achieve long-term sustained weight loss with lifestyle modification alone. Pharmacologic weight reducing agents in most patients are disappointing as they result in only modest short-term weight loss with significant side-effects and are expensive. Multiple systematic reviews and meta-analyses report the overwhelming benefit of metabolic surgery for weight loss and resolution of comorbid conditions when compared to non-surgical options. ${ }^{7-9}$ The body mass index (BMI) level at which metabolic surgery should be recommended has been vigorously debated. There is widespread consensus that surgery should be offered to those with a BMI $\geq 40 \mathrm{~kg} / \mathrm{m}^{2}$ or a BMI $\geq 35 \mathrm{~kg} / \mathrm{m}^{2}$ with associated comorbidity (such as diabetes, hypertension and coronary artery disease) and those who have not managed to achieve durable weight loss by other means. Much of the debate has centred around the BMI category of $25.0-34.9 \mathrm{~kg} / \mathrm{m}^{2}$ with some suggesting considering metabolic surgery in those with type 2 diabetes with a BMI $30.0-34.9 \mathrm{~kg} / \mathrm{m}^{2}$, however, few would suggest metabolic surgery for a BMI $<30 \mathrm{~kg} / \mathrm{m}^{2}{ }^{10}$

Several surgical studies have shown the benefit of surgery for sustained weight loss. Gloy et al. reported that bariatric procedures resulted in a mean difference of $26 \mathrm{~kg}$ in weight loss, as well as greater remission of diabetes and the metabolic syndrome and improvements in quality of life when compared to non-surgical treatment. ${ }^{9}$ Interestingly, they failed to show a difference between the various surgical procedures. Chang et al. showed that, after 5 years, bariatric surgical procedures resulted in a lowering of body mass index by $12-17 \mathrm{~kg} / \mathrm{m}^{2}{ }^{11}$ In their meta-analysis, gastric bypass resulted in greater weight loss but caused more complications, whereas adjustable gastric banding was safer but had a higher re-operation rate and resulted in less weight loss than gastric bypass. Similarly, a systematic review and meta-analysis by O'Brien et al. showed that in studies with $>10$ years of data gastric bypass resulted in a weighted mean of $56.7 \%$ excess weight loss (EWL) and laparoscopic adjustable gastric banding resulted in a $45.9 \%$ EWL. ${ }^{12}$ Therefore, metabolic surgery has become the mainstay of treatment for achieving long-term significant weight loss, with the laparoscopic Roux-en-Y bypass procedure considered the gold standard. In this issue of the journal, Dewar et al. report on a single centre's experience in the private sector in South Africa with performing laparoscopic adjustable gastric banding (LAGB). ${ }^{1}$ After a mean duration of follow-up of 39.1 months (range 3-86 months) they achieved $\mathrm{a} \geq 40 \%$ EWL in $66.3 \%$ of patients, with most of the weight loss occurring in the first year of follow-up. Furthermore, they documented resolution of type 2 diabetes in $56.4 \%$ of patients and resolution of prediabetes in $89.8 \%$ of patients. Overall, their surgical procedure was safe with only one band erosion but band slippage that occurred in $11 \%$, necessitating surgery for removal in $9 \%$ of patients. Their results are consistent with the literature showing safety and significant weight loss with resolution of comorbidity in the short- to mid-term. In the early 2000s, after FDA approval in 2001, LAGB was the most popular surgical procedure for obesity because of its ease of placement, reversibility, relatively low early mortality and complication rate, and 
its ability to achieve durable weight loss. However, with longer term follow-up it was soon noted that the reoperation rate, mainly for band erosion or band slippage, was higher and the amount of weight loss was less when compared to other surgical procedures..$^{11}$ It is true that LAGB has a higher reoperation rate than other bariatric procedures, however, it depends largely on surgeon experience ${ }^{13}$ and although the long-term weight loss achieved is less than other metabolic surgical procedures it remains significant and more than can be achieved with non-surgical methods. ${ }^{8}$ Whether to retain LAGB as a commonly performed surgical procedure for the management of obesity remains controversial because of the disparate results of studies. Studies with new generation bands and experienced surgeons are reporting long-term weight loss approaching 45-54\% EWL, similar to that obtained with the Roux-en-Y procedure. ${ }^{14}$ Recently, O'Brien et al. reported significant weight loss of $30.1 \mathrm{~kg}, 48.9 \% \mathrm{EWL}$ in 35 patients with LAGB that were followed for at least 20 years. Furthermore, their experience shows a decrease in reoperation rates with improving bands, greater surgical experience and better surgical aftercare. Poor aftercare was identified in this study as a risk factor for EWL emphasising the need to focus on mechanisms to improve this.

Since obesity has a plausible pathophysiology, a morbidity, a mortality, various treatment options and an improved outcome with treatment, it must now be considered a chronic disease. Thus far, metabolic surgery has been the only intervention to show longterm significant weight loss with resolution of obesity-associated comorbidity such as type 2 diabetes, hypertension and obstructive sleep apnoea. All metabolic surgery procedures have advantages and disadvantages. More studies are needed in low- and middle-income countries (LMICs) to show which metabolic surgery procedure would provide the best balance between cost-effectiveness and outcome, as the burden of disease and scarce resources preclude the gold standard Roux-en-Y procedure from being performed in the majority. In South Africa, metabolic surgery is largely confined to the private sector, but the outcomes have only been published in two reports. ${ }^{15,16}$ Over the past few years, more public sector academic units have started metabolic surgery programs. ${ }^{17,18}$ The public sector program needs to be extended, but re-source limitation and burden of disease will necessitate specific indications that focus on the best surgical option for those most likely to benefit, with surgery for the super obese centralised to minimise the risk of life threatening complications.

Finally, metabolic surgery will not address the primary drivers for obesity, this will require a multisectoral approach to prevention and identification of groups most at risk so that prevention programs can be focussed to the greatest need.

\section{REFERENCES}

1. Dewar GA, Urry RJ, Clifford SD, et al. Bioring ${ }^{\circledR}$ gastric banding for obesity in a private South African hospital. SAJS. 2020;58(3):115-121.
2. Chooi YC, Ding C, Magkos F. The epidemiology of obesity. Metabolism. 2019;92:6-10.

3. Shisana OL, Labadarios D, Rehle T, et al. SANHANES-1 Team. The South African National Health and Nutrition Examination Survey, 2012: SANHANES-1: the health and nutritional status of the nation. 2014 (cited $2020 \mathrm{Feb} 25$ ). Available from: http://hdl.handle.net/20.500.11910/2864.

4. National Department of Health, SSA, South African Medical Research Council, ICF. South Africa Demographic and Health Survey 2016. Pretoria, South Africa, and Rockville, Maryland, USA; 2019.

5. Apovian CM, Aronne LJ, Bessesen DH, et al. Pharmacological management of obesity: an endocrine society clinical practice guideline. J Clin Endocrinol Metab. 2015;100(2):342-62.

6. Prospective Studies Collabration. Body-mass index and causespecific mortality in 900000 adults: collaborative analyses of 57 prospective studies. Lancet. 2009;373(9669):1083-96.

7. Schauer PR, Kashyap SR, Wolski K, et al. Bariatric surgery versus intensive medical therapy in obese patients with diabetes. N Engl J Med. 2012;366(17):1567-76.

8. Buchwald H, Buchwald JN. Metabolic (bariatric and nonbariatric) surgery for type 2 diabetes: a personal perspective review. Diabetes Care. 2019;42(2):331-340.

9. Gloy VL, Briel M, Bhatt DL, et al. Bariatric surgery versus non-surgical treatment for obesity: a systematic review and meta-analysis of randomised controlled trials. BMJ. 2013;347:f5934.

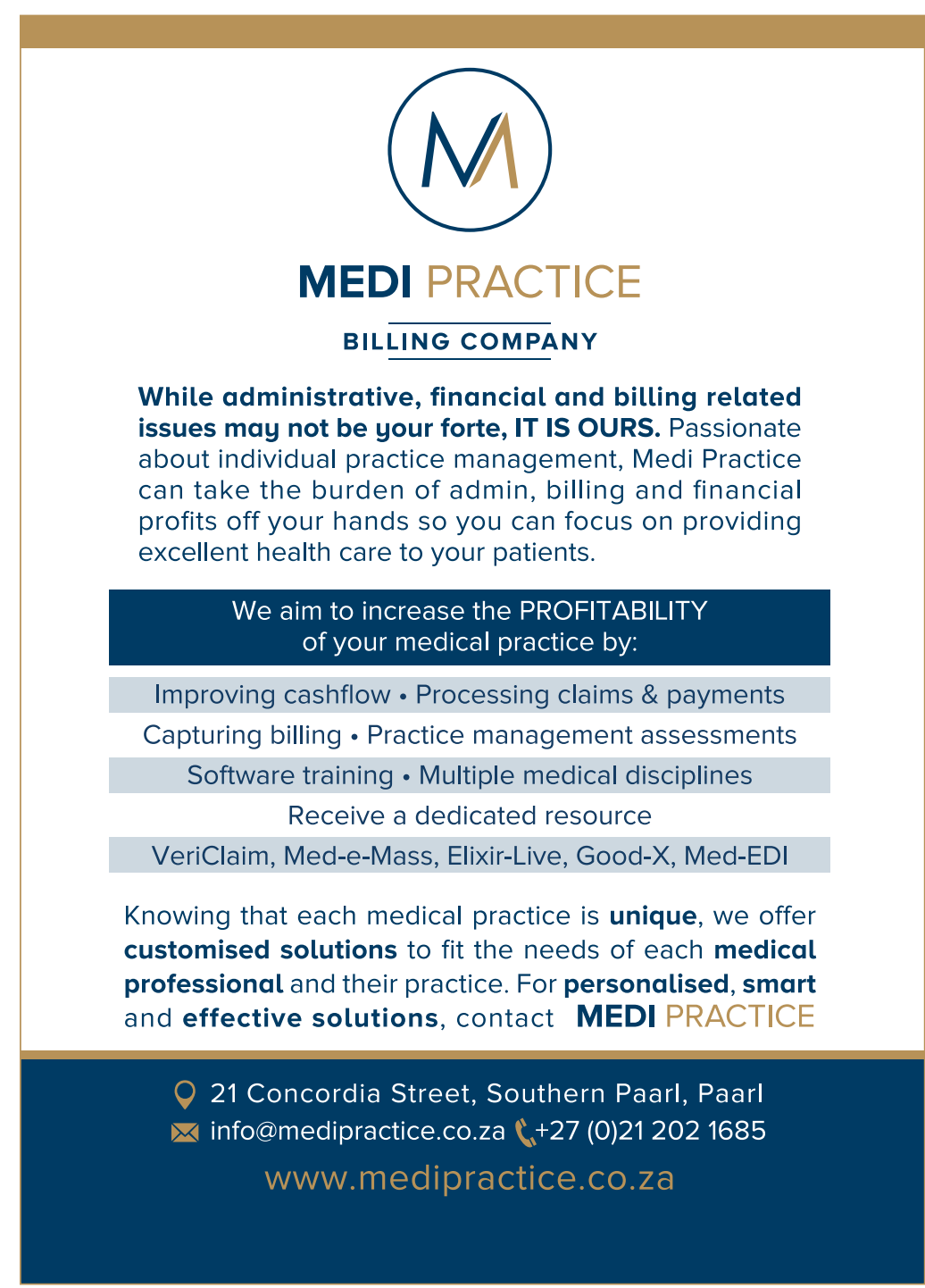


10. Semlitsch T, Stigler FL, Jeitler K, Horvath K, Siebenhofer A. Management of overweight and obesity in primary care - a systematic overview of international evidence-based guidelines. Obes Rev. 2019;20(9):1218-30.

11. Chang SH, Stoll C, Song J, et al. The effectiveness and risks of bariatric surgery: an updated systematic review and metaanalysis, 2003-2012. JAMA Surg. 2014;149(3):275-87.

12. O'Brien PE, Hindle A, Brennan L, et al. Long-term outcomes after bariatric surgery: a systematic review and metaanalysis of weight loss at 10 or more years for all bariatric procedures and a single-centre review of 20-year outcomes after adjustable gastric banding. Obes Surg. 2019;29(1):3-14.

13. Egberts K, Brown WA, O'Brien PE. Systematic review of erosion after laparoscopic adjustable gastric banding. Obes Surg. 2011;21(8):1272-9.

14. O'Brien PE, MacDonald L, Anderson M, Brennan L, Brown W. Long-term outcomes after bariatric surgery: fifteen-year follow-up of adjustable gastric banding and a systematic review of the bariatric surgical literature. Ann Surg. 2013;257(1):87-94.

15. Van der Merwe M-T, Fetter G, Naidoo S, et al, Baseline patient profiling and three- year outcome data after metabolic surgery at a South African centre of excellence. JEMDSA. 2015;20(3):37-42.

16. Sofianos C, Sofianos C. Outcomes of laparoscopic sleeve gastrectomy at a bariatric unit in South Africa. Ann Med Surg. 2016;12:37-42.

17. Lubbe J, Webner A, Potgieter A, et al. Metabolic surgery in South Africa: an initial academic hospital experience. SAJS. 2019;57(2):20-6.

18. Loots E, Sartorius B, Paruk IM, Clarke DL. The successful implementation of a modified enhanced recovery after surgery (ERAS) program for bariatric surgery in a South African teaching hospital. Surg Laparosc Endosc Percutan Tech. 2018;28(1):26-9. https://doi.org/10.1097/ SLE.0000000000000488. 